\title{
Sechs neue Käfer aus Deutsch Neu-Guinea.
}

\author{
Beschrieben von
}

Dr. K. M. Heller in Dresden.

\section{Scarabaeidae.}

Chalcocrates gen. nov. Cyclocephalidarum.

Mentum attenuatum, apice truncatum, galea validis, longe penicillata, intus acute dentata. Mandibulae acutae, resimae, maris superne visibiles, feminae obtectae breviores. Clypeus trapeziformis, margine antico reflexo, sutura clypeali solum utrinque observanda. Antennae deciens-articulatae, flabellum tri-articulatum. Pronotum transversum, marginatum, margine antico corneolimbato. Elytra estriata, ampliata, filamentoso-marginata. Pygidium verticale, elytris dimidia parte obtectum, haud recurvum. Processus prosternalis verticalis, apice oblique truncalus, antice paulo inter coxas antrorsum productus, postice parce ciliatus. Episterna metathoracis sat angulata (ut in genere Oryctoderus). Segmenta abdominalia inter se aequilonga, ultimum feminae in margine ciliato, apice sinuato. Tibiae anticae in utroque sexu extus tridentatae (dente apicali computato) intermediae posticaeque subteretes, haud prismaticae, tarsis anticis in femina vix, in mare tibiis anticis distincte longioribus; unguiculi omnes simplici, onychio manifesto, apice penicillato.

Kopfschild quer, trapezförmig, am Vorderrande schmal aufgebogen und daselbst in der Mitte leicht ausgerandet, beiderseits an der Basis (namentlich beim $\sigma^{7}$ ) durch eine undeutliche Naht abgesetzt. Kiefer sichtbar nach oben gebogen, beim Weibchen von oben nicht, beim Männchen aber deutlich über das Kopfschild vorragend, die Maxillen innen mit zwei (oder mehreren?) spitzen langen Zähnen bewehrt und besonders beim Männchen aufsen mit langem Haarpinsel besetzt. Kinn mindestens doppelt so lang wie an der Basis breit, nach vorn verschmälert und verrundet-abgestutzt, nur am Seitenrande punktirt und lang bewimpert. Fühler zehngliedrig, in beiden Geschlechtern gleichförmig, ihr 1. Glied ungefähr so lang wie der Fächer, keulenförmig, an der Wurzel stark verjüngt, das 2. oberseits an der Wurzel breit ausgerandet, das 3. Glied etwas länger als breit, das vierte sehr wenig breiter als lang, die folgenden Glieder quer. Prosternalfortsatz vertikal, stumpfwinklig nach vorn umgebogen (ähnlich wie bei Augoderia) und mit dem umgebogenen

\footnotetext{
Deustche Entomol. Zeitschr. 1903. Heft II.
} 
Theil sich zwischen und über die Vorderhüften einschiebend, nur am Hinterrande der Abschrägung spärlich bewimpert. Mittelbrust zwischen den Mittelhüften furchenartig eingeschnitten, Seitenstücke der Hinterbrust, wie bei Xylotrupes, ziemlich schmal, Afterdecke vertikal unten nicht eingezogen. Vorderschienen in beiden Geschlechtern dreizähnig (der Endzahn mitgezählt), Hinter- und Mittelschienen ungefähr halbcylindrisch, mit je zwei schrägen stacheltragenden Querleisten, von welchen die obere jedoch, namentlich an den Mittelschienen, undeutlich ist. Schienenspitze ohne Stachelkranz, von den üblichen zwei Endsporen ist der innere beim $q$ breit lamellenartig und zu einer scharfen Spitze abgeschrägt, beim $\sigma^{\top}$ einfach und fast doppelt so lang wie das erste Tarsenglied.

Die neue Gattung läfst sich in das gegenwärtige DynastidenSystem nicht ohne weiteres einreihen; sie nimmt unter den Cyclocephalen ebenso eine Ausnahmestellung ein, wie sie es unter den echten Dynastiden thun würde. Für eine Vereinigung mit letzteren sprechen die verlängerten Vordertarsen des Männchens, die aufgebogenen und wenigstens beim Mann von oben sichtbaren Mandibeln, sowie die Aehnlichkeit des Körperbaues mit Chalcosoma und Xylotrupes. Für eine Vereinigung mit den Cyclocephalen sprechen der Mangel einer Auszeichnung des männlichen Kopfes durch ein Horn (der übrigens auch bei Golofa inermis Thoms. beobachtet wird), die nicht kantigen, an der Spitze nicht erweiterten Hinterschienen und der breite Clypeus. Da die Cyclocephalen nach dem heutigen Stande der Systematik ohnedies aus sehr heterogenen Gattungen zusammengewürfelt erscheinen, bielt ich es für weniger bedenklich Chalcocrates unter diese einzureihen, als unter den echten Dynastiden für sie eine Ausnahmestellung zu erheischen, in der Ueberzeugung, dafs ein späterer Revisor des Dynastiden-Systems die Cyclocephalen auflösen oder doch in an. derer Weise abgrenzen müsse, als dies von Burmeister und Lacordaire geschehen ist.

\section{Chalcocrates Felschei sp. n. đㅇ․}

Capite, prothorace scutelloque obscure cupreis, opacis, elytris atropurpureis, singulis vittis tribus (exteriori marginali) luteotomentosis, apice, intermedia etiam basi, confluentibus; clypeo transverso-trapeziforme, maris disco vix perspicue subbinoduloso, subtiliter reticulato-aciculato ac interpunctato; prothorace dupliciter, creberrime subtileque ac fortius remoteque punctato, punctis majoribus umbilicatis; elytris adiposis, subtile remoteque punctatis, vitta 
denudata suturali postice attenuata, discoidali in humeros incipiente et usque in callo apicali extensa et hic dilatata, marginali antice posticeque abbreviata; epipleuris nigris aut piceis; corpore subter in mare fuscescenti-nigro-parum, praesertim in metasterno, aeneolavato, metasterno cum episternis subtiliter reticulatis ac interpunctatis, parce rufo piloso, segmentis abdominalibus nudis, in lateribus subtilissime ac creberrime reticulato-punctatis, in parte mediano fere laevibus. Tibiis purpureo-cuprascentibus in utroque sexu fere conformibus, tarsis nigris.

Long. tot. 37,5 (ㅇ 37), elytror. 25,5 (우 26,5), thoracis in linea mediana 10,5 ( $(11)$ mill.; lat. max. elytror. 20.5 (ㅇ 21), thoracis $15,5$ (ㅇ 15,8$)$ mill.

Patria: Nova Guinea germanica et britannica: Sattelberg, $\sigma^{\uparrow}$ coll. Prof. Hauser, Erlangen, et Moroka altitudine 2000 ped. leg. I 1896 Anthony i. coll. C. Felsche (ex coll. Rothschild).

Kopf, Halsschild und Schildchen schwärzlich kupferfarben, infolge der äufserst dichten und feinen Grundpunktirung matt; Clypeus quer trapezförmig, am Vorderrande schmal aufgebogen und daselbst in der Mitte leicht stumpfwinkelig ausgeschnitten, so wie der Kopf mit feinen netzartigen Nadelrissen und zwischen diesen punktirt. Form des Halsschildes ähnlich wie bei Xylotrupes gideon ㅇ, doch etwas flacher und kürzer, die Seiten mehr gerundet, mit der gröfsten Breite in der Mitte, die Vorderecken stumpf rechtwinkelig, oberseits nicht sebr gleichmäfsig und entfernt mit genabelten Punkten bedeckt. Flügeldecken schwärzlich purpurn, matt fettglänzend, mit fadenartigem Randsaum (wie bei Xylotrupes 오), schwachen Schulterbeulen und hinter der Mitte erweitert, neben der kaum wahrnehmbaren dichten und feinen Grundpunktirung überall, auch in den Tomentstreifen, fein und weitläufig punktirt. Die drei lehmgelben Tomentstreifen, von welchen der äufsere den Seitenrand einnimmt und hinten mit dem mittleren zusammenfliefst, verlaufen derartig, dafs ein gemeinsamer schwarzer, nach binten sich verschmälernder kahler Suturalstreifen, der an der Basis dreimal so breit ist wie das Schildchen, im Spitzendrittel plötzlich abgebrochen oder nur auf einen schmalen Sutaralstreifen reducirt erscheint, gebildet wird, dem, durch den hellen Tomentstreifen getrennt, nach aufsen der mittlere glatte Deckenstreifen folgt, der von der Schulter- bis zur Endbeule verläuft; er erreicht ebenso wenig den Deckenrand wie der äufserste kahle Längsstreifen, der vorn und hinten stark abgekürzt ist. 
Die beiden mir vorliegenden Stücke gehöreu verschiedenen Geschlechtern aus ziemlich weit von einander entfernten Fundorten an, doch besteht kaum ein $\mathrm{Z}$ weifel, dafs sie ein und derselben Art angehören, die geringfügigen Unterschiede sind als secundäre Geschlechtscharaktere anzusehen. So ist das $\sigma^{\tau}$ lebhafter in der Färbung, die Epipleuren sind schwarz, die Unterseite theilweise erzgrün angelaufen, während beim 우 Unterseite und Epipleuren dunkel rothbraun sind. Das Kopfschild des $\sigma^{\tau}$ ist etwas weniges länger und besitzt fast gerade Seitenränder im Vergleich zum 오, bei dem letztere leicht gebogen sind. Aufserdem ist die abgeschrägte Fläche des Prosternalfortsatzes beim $\sigma^{\lambda}$ etwas breiter, vorn gerade abgestutzt und im Gegensatz zum 오 mit einem deutlichen eiförmigen Eindruck versehen. Der dunkelbraune Forceps ist purpurn und grünlich erzfarben angelaufen; die Parameren besitzen aufsen einen stumpfwinkeligen flügelartigen Ansatz, sodals der Umrifs des Forceps dem eines stumpfen Pfeiles ähnelt.

\section{Lomapterairidescens sp. n. ऽ우.}

Ex affinitate semicastaneae Kraatz, colore variabile, aut rufa, aut rufa ac nigro-signata, aut nigra, aut nigra femoribus tibiisque, partim aut totis, rufis, supra paulo iridescens; elytris ad basin multo parcius transverse-strigosis, corpore subter fulvo-setoso.

Mas forcipe robusto (multo latiore quam in albertisi), fibulis medianis, corneo-chitinosis, a basi divergentibus.

Femina pygidio semiovale-producto, super pone suturae apicem subcalloso, subter carina longitudinali, in dimidia parte basali intruso ac longitudinaliter subtiliterque strigoso, margine baseo-ventrale medio in lamina transversu, circiter longitudine quadruplo latiore dilatato, laminae margine antico sinuato, segmento quinto medio haud sinuato. - Long. 25-27 mill., lat. 13-14 mill.

Patria: Nova Guinea germanica: Sattelberg, leg. C. Wahnes et in coll. Prof. G. Hauser, Erlangen.

Diese zu dem nicht metallischen Artenkreis gehörige neue Lomaptera-Form zeigt die Decken im Basaltheil viel sparsamer und kürzer quergestrichelt, wodurch sie sich sofort von der viel gracileren pallens (die bis zur Wurzel hin strigilirte Decken hat) und ebenso von semicastanea und negata (erstere mit schwarzer, letztere mit röthlich-gelber Unterseiten-Beborstung), deren Deckenbasis ganz glatt ist, unterscheidet. Sie ähnelt in der Deckenskulptur am meisten der L. rufa Kraatz, unterscheidet sich aber im männl. Geschlecht sehr scharf durch die Form des Forceps, 
beim Weibchen durch die Form des Pygidiums. Die Färbung ist aufserordentlich veränderlich, entweder röthlich gelbbraun, mit theilweise oder ganz schwarzem Kopf und Unterseite, häufig sind dann auch die Deckenbasis und Schulter und die Beine schwarz, oder der ganze Käfer ist tief schwarz, oder schwarz mit rothgelben Hinter- und Mittel-Schenkeln und -Schienen. Selten nur gleichen sich zwei Stücke vollkommen.

Kopf ähnlich wie bei $L$. rufa, mit fast glatter Stirn. Halsschild fein und zerstreut (beim $\sigma^{\uparrow}$ gröber), an den Seiten stärker punktirt, die Punkte daselbst kurze Bogen darstellend, ähnlich wie bei semicastanea $\mathrm{Kr}$., aber nirgends mit derartigen queren Nadelrissen wie rufa Kr. Decken mit glatten Endschwielen, Naht in der hinteren Hälfte erhaben, ihre Spitze etwas vorgezogen. Forceps des Männchens absolut grölser als von der gröfseren L. Albertisi, unsymmetrisch, die Spitze auf der rechten Seite länger ausgezogen, die Parameren höchstens doppelt so lang als an der Basis breit, ihr Umrifs ungefähr elliptisch (bei L. rufa $\mathrm{Kr}$. sind die Parameren fast ihrer ganzen Länge nach zu einer schmalen linearen Platte verschmolzen). Die bei $L$. Albertisi, limbifera und rufa (vielleicht auch bei semicastanea?) häutigen Mittelspangen sind bei iridescens dunkelbraun, stark chitinisirt und von der Wurzel aus gespalten (bei Albertisi sind sie erst hinter, bei rufa in und bei limbifera vor der Mitte gegabelt). Pygidium des Weibchens in Form einer halben Ellipse vorgezogen, oberseits in der Mitte der Basis (unterhalb der Nahtspitze) mit einer höckerartigen Schwiele, unterseits mit fast vertikaler Basal- und horizontaler Apicalhälfte, in der Mitte mit einem Längskiel und an der Basis sehr fein längsnadelrissig, die mittlere plattenartige Erweiterung des Basalrandes stark quer, circa viermal so breit wie lang, vorn im flachen Bogen ausgerandet. Bauchringe in der Mitte mit einer Querreihe gelblicher Borsten. Vordertarsen ohne Längsnadelrisse, einfach fein und zerstreut punktirt, ihr Aufsenrand beim $\sigma^{\uparrow}$ ohne, beim $q$ mit kleinem stumpfen Zahn.

\section{Curculionidae.}

Autillia gen. nov. Ithyporidarum.

Rostrum elongatum, subarcuatum, apice depressiusculum ac paulo dilatatum, metasternum attingens, scrobes laterales, postice haud confluentes. Oculi fere rotundati, post circumsulcati. Antennae scapo oculos haud attingente, ante medium insertae, funiculo 7-arti- 
culato, articulis duabus primis elongatis, reliquis longitudine decrescentibus, septimo transverso, clava sat lata, subconica apice rectangulariter acuminata. Prothorax oblongus, lobis ocularibus manifestis. Mesosternum sat latum, horizontale, antice rectangulariter declive, utrinque margine granuloso-uncato ac ciliato. Scutellum oblongum. Elytra basi truncata, prothorace latiora, cuneiformes, deciens-striata. Segmentum primum abdominale, pulvinatum, duobus sequentibus unitis aequilongum. Coxae anticae valde distantes. Metasternum inter coxas intermedias et posticas coxarum diametro haud longiore. Femora sublineares, edentata, postica ultra elytrorum apicem protensa. Tibiae rectae, subcompressae, apice unco valido, posticae corbiculis apertis. Unguiculi liberi.

Nach Lacordaires Gattungstabelle (Genera des Coléoptéres VII, p. 50-51) würde man bei dem Versuch, die neue Gattung einzureihen, auf die Gruppe der Strongylopteridae stofsen, doch hat sie mit dieser nichts gemein; vor Allem trennt sie ihr langer, schlanker, an Palaeocorynus Faust und Haplocorynus Faust erinnernder Rüssel von dieser Gruppe. J. Faust bat (Stett. Ent. Ztg. 1893, p. 232-234) die afrikanischen Ithyporiden nach anderen Merkmalen tabellarisch zusammengestellt und daselbst die Gattung Amblyochetus aus Madagaskar charakterisirt. So wie diese unter den Afrikanern die einzige Gattung mit linearen Schienen ist, so bildet Autillia unter den indo-australischen Ithyporiden mit ihren linearen Schenkeln und Schienen eine Ausnahme. Sie dürfte, obgleich ihre Fühlerfurche der ganzen Länge nach von der Seite sichtbar ist, am besten unter die "Ithyporides vrais" einzureihen und in die Nähe von Colobodes zu stellen sein, von dem sie sich vor allem durch die Beine und die breite fast gleichseitig dreieckige Mesosternalplatte unterscheidet.

Die einzige bisher bekannte, aber sehr auffallende Art ist:

\section{Autillia horridipes sp. n.}

Nigra, glabra, elytris plagis rotundatis quatuor majoribus croceo-sericato-squamosis; prothorace utrinque in margine antico pedibusque rigide nigro-setosis; rostro basi crebre punctulato, parce ac minutissime margaritaceo-squamulato, fronte inter oculos puncto intruso, capite sat fortiter, prothorace aliquid inaequaliter punctato, laleribus punctis remotiis seriaque subtile e squamulis margaritaceis; elytris striato-punctatis, basi resimis, humeris transverse rugosis, singulis plagis convexis croceis duabus, una ante, altera pone medium, hac sutura propiore; pedibus nigris, plus minusve cyanescenti- 
bus, nigro-, tibiis anticis praeterea albido-setosis; tarsis super albotomentosis ac nigro-setosis.

Long. (vertice ad elytr. apicem) 10,5 - 12 mill., lat. ad hum. 4,5-5 mill.

Patria: Nova Guinea germanica: Sattelberg, legit. C. Wahnes, etiam i. coll. Prof. G. Hauser.

Schwarz, die Beine, namentlich die Schienen mehr oder weniger stahlblau angelaufen. Rüssel in der Apicalbälfte äufserst fein und weitläufig, an der Basis dicht und gröber punktirt und daselbst mit undeutlichem Mittelkiel und spärlich mit kleinen grünlichen Schuppchen bedeckt. Halsschild länger als breit, seine gröfste Breite vor der Mitte, vorn halsartig abgesetzt und der Vorderrand etwas vorgezogen; Hinterrand fast gerade abgestutzt, oberseits ziemlich dicht, an den Seiten sehr weitläufig und ungleich grofs punktirt, beiderseits in der Nähe der Augenlappen mit kurzen, steifen, nach vorn gerichteten schwarzen Borsten und bei gut erbaltenen Exemplaren aufserdem mit einer vom oberen Augenlappenrand nach den Halsschildhinterecken laufenden einfachen Reihe von kleinen grünlichen Schüppchen. Flügeldecken etwas länger als $1 \frac{1}{2} \mathrm{mal}$ so lang wie breit, hoch gewölbt, Scheitel der Wölbnng vor der Mitte und nach vorn steiler als nach hinten abfallend. Wurzelrand der Decken wulstartig aufgeworfen. Schultern etwas beulenartig abgesetzt und querrunzelig. Spatien zwischen den kräftigen Punktreihen äufserst fein und zerstreut punktirt, die Naht aufserdem, besonders im abschüssigen Theil, sehr fein längsrunzelig, jede Decke mit je einer runden gewölbten Makel von Augengrölse vor und hinter der Mitte, die vordere tangirt innen den 3., aufsen den 6., die hintere den 1. und 3. Punktstreifen; diese Pusteln, die zufolge ihrer Stellung an die von Pachyrhynchidius IVpustulatus Faust (Stett. E. Z. LVIII, 239) erinnern, sind mit seidenartig glänzenden linearen, federartig sich überdeckenden rothgelben Schuppchen bedeckt. (Bei mikroskopischer Vergröfserung weisen sich diese Schüppchen mit Ausnahme ihrer knötchenartig verdickten Spitze längsgestreift aus.) Die Punkte der Deckenstreifen auf ihrem Grunde mit je einem sehr kleinen grünlich oder selbst roth schimmernden Schüppchen. Seiten des Körpers, Schenkel und Schienen sparsam mit grünlichen Schüppchen bedeckt, die beiden letzteren mälsig dicht mit schräg abstehenden schwarzen Borsten bestanden, Vorder- und Hinterrand namentlich der vorderen Schienen aufserdem mit weifslichen Wimpern besetzt. Tarsen oberseits weifs tomentirt, sparsam schwarz beborstet. 
Unter den mir vorliegenden 3 Exemplaren befindet sich eines, bei dem nicht nur die hintere rechte Schuppen-Makel in drei verschieden grofse getheilt erscheint, sondern bei dem aufserdem noch auf dem Spatium vor der Mitte 1, auf der rechten Decke 2, auf der linken 3 kleine accessorische Schuppenmakeln vorhanden sind. Die grölseren Weibchen scheinen sich dadurch von den Männchen zu unterscheiden, dafs ihr 2 tes Bauchsegment der ganzen Quere nach tief furchenartig eingedrückt ist.

\section{Talanthia papuana sp. $\mathrm{n}$.}

T. phalangio Pasc. simillima sed major; thorace linea mediana alba, medio interrupta; elytris singulis vitta alba, in scutello incipiente, extrorsum paulo curvata et ante elytrorum medium striam secundam attingente, ante apicem macula transversa et in humeris macula minore albo-squamosis, sutura spatioque secundo pone medium elevatis, sutura usque ad apicem granulis, aspersis, seriatis, granulis in spatio secundo eadem magnitudine.

Long. (rostro non computato) 11 mill.

Patria: Nova Guinea Germanica: Sattelberg, in coll. Prof. G. Hauser, Erlangen.

Die von Penang beschriebene, aber wohl auch auf Sumatra und Java vorkommende T. phalangium Pasc. (Ann. Mag. Nat. Hist. 1871 , p. 207, Pl. XV, fig. 4) besitzt aufser der Scutellarmakel auf jeder Decke noch 3 Makeln, während bei papuana die Scutellarmakel durch einen nach aufsen gekrümmten Längsstreifen ersetzt ist, den man sich durch eine Verschmelzung des Scutellarfleckens mit der mittleren Makel bei phalangium entstanden denken kann; die Apicalmakel fehlt bei papuana ganz, die Subapicalmakel ist quer rechteckig. Von Sculpturunterschieden ist nur zu erwähnen, dafs die Naht und das 2 te Spatium hinter der Mitte etwas gewölbt vortreten und dafs erstere mit scharfen Raspelzähnchen bis zur Spitze hin besetzt sind und dafs die Körnchen des 2 ten Spatiums nicht kleiner als die der Naht sind. Alles übrige stimmt mit T. phalangium überein.

\section{Asytesta ypsilon sp. n.}

Nigra, antennis rufis, super fuliginoso-velutina, lateribus capiteque cervino-squamosis, fronte nigro-bimaculata, thorace linea ochraceo-albida, parabolica, margine antico angulisque posticis tangente, in elytris continuatis et ad suturam convergentibus, hanc ante medium attingente, de hic sutura usque ad apicem sequente; rostro basi tri- 
Dr. K. M. Heller: Sechs neue Käfer aus Deutsch Neu-Guinea. 313.

carinulato; prothorace basin versus angustato, disco remote ac subtiliter, lateribus manifeste granulatis; elytris substriatis, sutura basi impressa, spatiis, secundo excepto, seriato-granulatis, in lateribus granulis multo majoribus; femoribus squamulis albis adspersis, tibiis haud compressis, tarsis articulo ultimo unguiculisque rufis.

Long. 6,5, lat. 3 mill.

Patria: Nova Guinea germanica: Sattelberg, i. coll. Prof. G. Hauser, Erlangen.

Von der Gröfse der A. gazella Oliv., oberseits sammetartig nufsbraun, an den Seiten rehbraun beschuppt, eine gelblichweifse Binde von Parabelform, deren Scheitel mit dem Halsschildvorderrande zusammenfällt und deren Schenkel die Halsschildhinterecken schneiden, setzt sich an den Schultern auf die Flgd. fort und trifft, leicht einwärts gekrümmt, vor der Mitte der Naht auf diese, die von da ab bis zur Spitze gelblich weifs beschuppt ist, sodafs die Decken eine Y-ähnliche Figur aufweisen. Rüssel an der Wurzel mit drei abgekürzten Längsleisten, Stirn rehbraun beschuppt, mit zwei schwärzlichen Makeln. Halsschild so lang wie breit, vor der Mitte am breitesten, Scheibe mit unregelmäfsig vertheilten kleinen Körnchen sparsam bedeckt, Halsschildseiten mit groben abgeschliffenen Körnern bedeckt. Die dunkelbraune Färbung der Halsschildoberseite greift auf die Halsschildseiten über und bildet unterhalb der weifslichen Randlinie eine halbelliptische Makel. Flügeldecken undeutlich gestreift, Naht an der Wurzel eingedrückt, Spatien, mit Ausnahme des zweiten, mit Reihen entfernter Körner, die vom 5 ten Spatium nach aufsen hin gröfser als auf den inneren Spatien sind. Die Reihe auf dem dritten Spatium ist hinten, die auf dem vierten vorn und hinten stark abgekürzt, sodafs letztere nur aus 4 Körnern besteht. Beine schwärzlich, die Schenkel mit zerstreuten, weilslichen Schüppchen, die Schienen nicht comprefs, innen (auf ihrer den Schenkeln zugekehrten Seite, bei den hinteren also auf der Vorderseite) mit vier Längsleisten. Hinterschenkel nur mäfsig die Deckenspitze überragend. Drittes und viertes Tarsenglied röthlich.

\section{Arachnopus patronoides sp. n.}

Arachnopo palrono Pasc. similis sed minor, antennis gracilioribus, prothorace linea mediana nulla, elytris apice fortius acuminatis, sutura basi impressiuscula albo-squamulata (basi eycepta), interstitiis fortius asperatis, tribus internis dimidia basali convexis, 
elytris singulis in ultimo triente linea mediana apicem attingente, prosterno impressione longitudinali in fundo albo-squamosa.

Long. 10, lat. 4,5 mill.

Patria: Nova Guinea Germanica: Sattelberg, i. coll. Prof. G. Hauser, Erlangen; etiam legit. C. Wahnes.

Die Art ähnelt infolge der grofsen, rundlichen, weifsbeschuppten Schultermakeln dem patronus Pasc. (der auch aufser den Makeln noch weilse Längslinien auf den Decken besitzt), A. binotatus Pasc. und fossulatus Faust, die auch grofse Schulterflecke haben, besitzen keine weifsen Streifen auf den Decken.

Rüssel an der Wurzel mit 5 abgekürzten Längsleisten, Stirn zwischen den Augen mit eingestochenem Punkt. Fühler näher der Rüsselwurzel als der Spitze eingefügt, der Schaft und die 2 ersten Geifselglieder röthlichbraun, die Geifsel (ähnlich wie bei gazella Ol.) viel schlanker als bei patronus, die Glieder an der Basis deutlich konisch verjüngt (bei palronus fast cylindrisch), das 2 te Geifselglied etwas länger als das 3 te und 4 te zusammengenommen, das 5 te kugelig, kaum so lang wie breit. Halsschild ziemlich dicht raspelartig gekörnelt, beiderseits mit zwei, in der Mitte ohne weifse Längslinien. Vorderbrust nicht wie bei patronus mit einer weifsen Querlinie, sondern der Länge nach breit eingedrückt und auf dem Grunde des Eindruckes weils beschuppt. Flügeldecken nur wenig länger als breit, eiförmig, hinten zugespitzt, 1.-3. Spatium in der Basalhälfte gewölbt, raspelartig gekörnelt, die Körnchen mit von hinten her eingestochener Borste und nach der Deckenspitze zu kleiner werdend. Ein grofser elliptischer Fleck an der Schulter, die Naht, ausgenommen an der Wurzel, ein Streifen in der Mitte des letzten Deckendrittels und der Deckenseitenrand in der hinteren Hälfte weifs beschuppt.

Charakteristisch für die Art ist auch die Deckenwölbung. Die Rückencontur zeigt bei seitlicher Ansicht ihre grölste Convexität vor der Deckenmitte, senkt sich dann allmählich nach abwärts und bildet vor der Spitze eine Knickung. 


\section{$2 \mathrm{BHL}$ Biodiversity Heritage Library}

Heller, K M. 1903. "Sechs neue Käfer aus Deutsch Neu-Guinea." Deutsche entomologische Zeitschrift 1903, 305-314. https://doi.org/10.1002/mmnd.48019030218.

View This Item Online: https://www.biodiversitylibrary.org/item/103315 DOI: https://doi.org/10.1002/mmnd.48019030218

Permalink: https://www.biodiversitylibrary.org/partpdf/101188

\section{Holding Institution}

Harvard University, Museum of Comparative Zoology, Ernst Mayr Library

\section{Sponsored by}

Harvard University, Museum of Comparative Zoology, Ernst Mayr Library

\section{Copyright \& Reuse}

Copyright Status: Public domain. The BHL considers that this work is no longer under copyright protection.

This document was created from content at the Biodiversity Heritage Library, the world's largest open access digital library for biodiversity literature and archives. Visit BHL at https://www.biodiversitylibrary.org. 